\title{
Millisecond pulsars at low frequencies
}

\author{
N. D. Ramesh Bhat ${ }^{1,2}$, Steven E. Tremblay ${ }^{1,2}$ and Franz Kirsten ${ }^{1}$ \\ ${ }^{1}$ International Centre for Radio Astronomy Research (ICRAR), Curtin University, \\ 1 Turner Avenue, Technology Park, Bentley, WA 6102, Australia \\ email: ramesh.bhat@curtin.edu.au \\ ${ }^{2}$ ARC Centre of Excellence for All-Sky Astrophysics (CAASTRO)
}

\begin{abstract}
Low-frequency pulsar observations are well suited for studying propagation effects caused by the interstellar medium (ISM). This is particularly important for millisecond pulsars (MSPs) that are part of high-precision timing applications such as pulsar timing arrays (PTA), which aim to detect nanoHertz gravitational waves. MSPs in the southern hemisphere will also be the prime targets for PTAs with the South African MeerKAT, and eventually with the SKA. The development of the Murchison Widefield Array (MWA) and the Engineering Development Array (EDA) brings excellent opportunities for low-frequency studies of MSPs in the southern hemisphere. They enable observations at frequencies from $50 \mathrm{MHz}$ to $300 \mathrm{MHz}$, and can be exploited for a wide range of studies relating to pulsar emission physics and probing the ISM.
\end{abstract}

Keywords. pulsars: general — pulsars: individual (PSR J0437-4715) — ISM: general

\section{Introduction}

The detection of a stochastic gravitational-wave background produced by supermassive black-hole mergers is the key science driver for current and future pulsar timing arrays (Janssen et al. 2015). With pulsar timing arrays (PTAs) around the world achieving sub-microsecond timing precision, it has become important to carefully assess various contributing factors to the noise budget in timing data, including e.g. the jitter noise arising from intrinsic pulsar emission, and interstellar delays and distortions caused by propagation effects. As ISM effects scale steeply with the observing frequency $\nu$ (e.g. dispersion $\sim \nu^{-2}$; pulse broadening $\sim \nu^{-4}$ ), low-frequency observations are most promising for a detailed characterisation of the ISM along the sight lines to PTA pulsars. Recent investigations suggest that compensating for ISM effects in PTA measurements, e.g. correcting for time-varying dispersion measure (DM), is more complicated than previously thought (e.g. Keith et al. 2013), and moreover, theoretical efforts highlight the importance of subtle effects such as chromatic DM (Cordes et al. 2016), and the need to devise optimal observing strategies for effective mitigation of ISM effects (Lam et al. 2015).

\section{Pulsar capabilities}

Over the past decade, a suite of new low-frequency arrays have become available for pulsar science; e.g. the Murchison Widefield Array (MWA), the Long Wavelength Array and Low Frequency Array; all operating at frequencies $\lesssim 300 \mathrm{MHz}$ and thus complementing well facilities such as the GMRT and the GBT that are sensitive at intermediate frequencies $(\approx 300 \mathrm{MHz}$ ). Originally conceived as an imaging instrument, the MWA is now geared up as a pulsar-capable facility, through the capture of fine-channelised voltage time series (Tremblay et al. 2015) and subsequent post-processing to form sensitive tied-array beams on the sky. In addition, we have implemented a pulsar capability for 

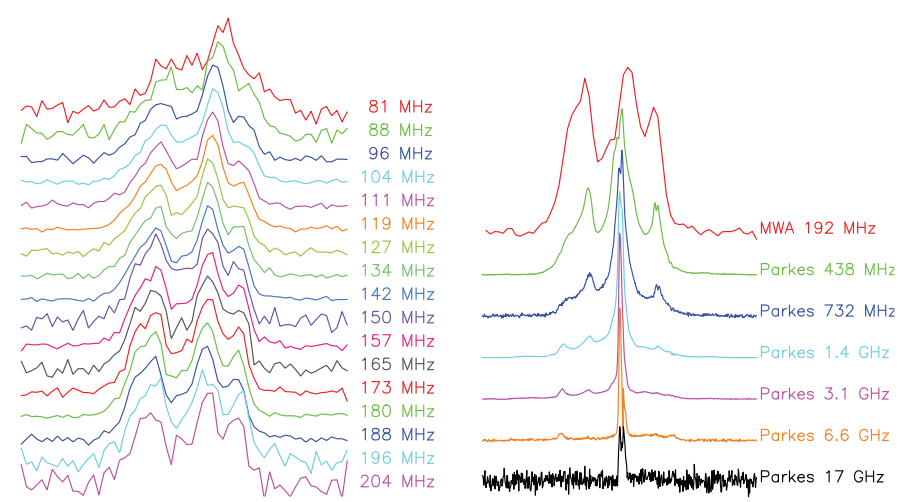

Figure 1. Integrated profiles of PSR J0437-4715 at frequencies from $\sim 80 \mathrm{MHz}$ to $\sim 17 \mathrm{GHz}$; data spanning multiple frequencies across the MWA band (left panel) were recorded simultaneously by distributing $30.72-\mathrm{MHz}$ VCS recording bandwidth into multiple units (i.e. sub-bands).

the Engineering Development Array (EDA; Wayth et al. 2017), a 35-metre station equivalent built from 256 MWA dipoles, primarily built for SKA prototyping and verification purposes. The EDA allows pulsar observations over a large instantaneous bandwidth of 50-300 MHz. These two facilities thus offer promising avenues for pulsar science that require simultaneous data at multiple frequencies or a large instantaneous bandwidth.

\section{PSR 0437-4715: a case study}

PSR J0437-4715 is an important object for PTAs; its brightness, relative proximity and superior timing precision all make it highly promising for wide-ranging applications including developing techniques (e.g. high-precision polarimetry). Our early observations with the MWA revealed a dramatic evolution of its pulse profile with frequency (Bhat et al. 2014), and more recently, our high-resolution dynamic spectral studies revealed parabolic scintillation arcs (Bhat et al. 2016). Using our feature extraction technique that yields a robust estimation of the curvature of the arc $(\eta)$, and the precisely-known three-dimensional sky geometry of the pulsar, we were able to determine the location of the underlying scattering screen as $115 \pm 3 \mathrm{pc}$, which is consistent with the distance to the edge of the Local Bubble ( 110-120 pc) in the direction of the pulsar.

The flexible design within the MWA's signal path allows simultaneous observations at multiple frequencies by distributing the $30.72-\mathrm{MHz}$ recording bandwidth into multiple chunks that span the $80-300 \mathrm{MHz}$ range (Fig. 1). Such observations enable a range of applications including studying the spectral evolution of the mean pulse shape, precision determination of DMs and the frequency scaling of scintillation properties. Further details on these aspects will be reported in a forthcoming publication (Bhat et al. in prep.)

\section{References}

Bhat, N. D. R., Ord, S. M., Tremblay, S. E., et al. 2014, ApJ, 791, L32

Bhat, N. D. R., Ord, S. M., Tremblay, S. E., McSweeney, S. J., \& Tingay, S. J. 2016, ApJ, 818, 86

Cordes, J. M., Shannon, R. M., \& Stinebring, D. R. 2016, ApJ, 817, 16

Keith, M. J., Coles, W., Shannon, R. M., et al. 2013, MNRAS, 429, 2161

Lam, M. T., Cordes, J. M., Chatterjee, S., et al. 2016, ApJ, 821, 66

Janssen, G., Hobbs, G., McLaughlin, M., et al. 2015, Advancing Astrophysics with the Square Kilometre Array (AASKA14), 37

Tremblay, S. E., Ord, S. M., Bhat, N. D. R., et al. 2015, PASA, 32, e005 